\title{
Potentially preventable readmissions - narrowing identification of patients at risk of unplanned readmission
}

\author{
M Berlinguet, ${ }^{*}$ L Licoppe, K Mitchell, O Weiser \\ From 26th Patient Classification Systems International (PCSI) Working Conference \\ Munich, Germany. 15-18 September 2010
}

\section{Introduction}

This aim of this study is to narrow patients truly at risk for avoidable readmissions, excluding the ones who are identified as planned readmissions.

The Belgian administrative dataset contains additional information not collected in the USA dataset, namely the 'planned' versus 'unplanned' admission status. Using this flag allows us to identify readmissions that are probably not avoidable, because they are medically justified (e.g., cholecystectomies delayed for anti-biotherapy), and to excluded them from calculation of potentially preventable readmissions (PPR).

\section{Methods}

We retain one fifth of all 2008 day-care and inpatient acute-care discharges from a representative sample of Belgium hospitals ( $n=496000$ or $19 / 113$ hospitals) that have agreed to take part in a comparative project, and who have provided a new data element - admission date which was unavailable before and is essential for the computation of PPRs.

All retained discharge abstracted data (DAD) were processed using the APR-DRG grouper included in the official DRG payment system in Belgium. Then, the PPR indicators are generated using specific software.

The output of the PPR-grouper is the creation of chains of readmissions over a 15-day selected interval, applying clinically defined inclusion and exclusion criteria, thus enabling identification of those readmissions that could be potentially avoided. This method is used in several US states. The state of Florida publishes statewide adjusted PPR rates for public hospitals, and we use

3M Health Information Systems, Wallingford, CT, USA its 2007 norm to risk adjust and compare our Belgian case-mix sample.

First, a comparison of the rates of PPR readmissions (without planned readmissions) is done in relation to all admissions, independent from any case-mix adjustment.

Second, indirect standardization rates for the same types of readmissions as above are computed using the Florida norm for PPR to adjust for case mix. Using indirect standardization, the Florida expected rates of PPR are applied on our number of discharges for each of 1300 combinations of DRG/severity sub-classes

\section{Results}

The observed number of at-risk stays (the number of stays remaining after initial exclusions) decreases by almost $25 \%$ when planned readmissions are excluded. The actual number of PPRs decreases two fold, from close to 2,500 PPRs (rate of 38 PPR per thousand stays) for all discharges to less than 1,250 PPRs (rate of 25 PPRs per thousand stays), when applied on stays that do not include planned readmissions.

Even more impressive, the Standardized Readmission Rate (SRR) using all stays is calculated at .9981, indicating .19\% PPR less in this sample than the Florida norm. However, when we use only the sample with no planned readmissions, we obtain a SRR of .679, indicating $32.1 \%$ fewer readmissions, a statistically significant (CI at 95\%) lower number of readmissions in relation to the Florida norm. There is also a statistically significant reduction of PPR using the sample with no planned readmissions in relation to all stays.

This difference of SSR from the Florida norm, excluding planned readmissions, is even more striking for medical DRGs (1.081 vs. .704) in relation to surgical DRGs (.898 vs. .6455). 
Our study indicates that the planned readmission status considerably improves the PPRs rating in Belgium, not only inside a single hospital, but also when compared to an external norm. Validation must be provided for accurate definition of planned readmission in Belgium and elsewhere. Also, validation of the linkage of various episodes of care must be ascertained.

\section{Conclusions}

Narrowing the number of patients at risk allows us to focus resources and prevention activities on those truly at need. So the study proves the usefulness of the PPR rate as an outcome indicator to monitor quality of care and outcome over time.

Published: 6 October 2010 